\title{
On Consistent Operators and Reflexivity
}

\author{
Edward A. Azoff, Wing Suet Li, Mostafa Mbekhta \\ and Marek Ptak
}

\begin{abstract}
We study Hilbert space operators $A=\oplus_{i \in \mathbb{N}} A_{i}$ which are consistent in the sense that each $A_{i+1}$ contains a copy of $A_{i}$. The formal definition is reminiscent of the classical ordering on projections in a von Neumann algebra. It is shown that if the powers of $A$ are simultaneously consistent, then $A$ must be reflexive. This is applied to study reflexivity of power partial isometries.
\end{abstract}

Mathematics Subject Classification (2010). Primary 47L05;

Secondary 47L45, 47L80.

Keywords. Reflexive algebra, direct sum of shifts, isometry, linear order, power partial isometry.

\section{Introduction}

An algebra of operators on a Hilbert space is said to be reflexive if its lattice of common invariant subspaces determines the algebra in the sense that any operator which leaves all the invariant subspaces for the algebra invariant has to belong to the algebra itself. An individual operator is reflexive in case the algebra it generates is reflexive. The concept has been studied extensively for nearly half a century, starting with Sarason [18] who established reflexivity of the unilateral shift. Reflexivity of isometries was then obtained by Deddens [10]. Deddens and Fillmore presented necessary and sufficient conditions for reflexivity of operators acting on finite-dimensional Hilbert spaces in [11].

An operator $T$ is said to be a power partial isometry if $T^{n}$ is a partial isometry for every positive integer $n$. These operators are all direct sums of members of the above classes and this paper examines their reflexivity. Direct sums of operator algebras are reflexive if and only if the same is true of all of their direct summands, but the situation is more complicated for direct sums of individual operators. Indeed, while it follows from [11] that direct sums of reflexive operators acting on finite-dimensional spaces remain reflexive, in [16], Larson and Wogen constructed a reflexive operator acting on $\ell_{2}$ whose direct sum with the zero operator fails to be reflexive.

The Deddens-Fillmore proof exploits the fact that smaller blocks appearing in the Jordan form of a nilpotent matrix are "corners" of the larger blocks. The concept of consistent operator formalizes this observation and 
provides a technique for proving that certain infinite direct sums of operators are reflexive.

The balance of the paper is organized as follows. Section 2 recalls some preliminary facts, focusing on the celebrated property $\left(\mathbb{A}_{1}\right)$ which has played a crucial role in invariant subspace theory. Consistent operators are introduced in Sect. 3. In Sect. 4, the consistency notion is first applied to infinite direct sums of Jordan blocks and then to general power partial isometries. The final section discusses connections to earlier work.

\section{Preliminaries}

Write $\mathcal{L}(H)$ for the algebra of bounded linear operators on a seperable Hilbert space $H$, and $\mathcal{F}(H)$ and $\tau c(H)$ for the ideals of finite rank and trace class operators in $\mathcal{L}(H)$ respectively. For $k \in \mathbb{N}$, write $\mathcal{F}_{k}(H)$ for the collection of operators having rank at most $k$. For $t \in \tau c(H)$, the trace norm of $t$ is $\|t\|_{1}=\operatorname{tr}\left(\left(t^{*} t\right)^{1 / 2}\right)=\sum_{n}\left(\left(t^{*} t\right)^{1 / 2} e_{n}, e_{n}\right)$, where $\left\{e_{n}\right\}$ is an orthonormal basis for $H$; the trace norm is independent of the choice of this basis. Reference to $H$ is suppressed when the underlying Hilbert space is clear.

It is fundamental that $\mathcal{L}(H)$ is the dual space of $\tau c(H)$ under the pairing $\langle A, t\rangle=\operatorname{tr}(A t)$ for $A \in \mathcal{L}(H)$ and $t \in \tau c(H)$. The ideals of finite rank and trace-class operators respectively induce the weak operator and weak* topologies on $\mathcal{L}(H)$. For any operator $A \in \mathcal{L}(H)$ we denote by $\mathcal{W}(A)$ (respectively, $\mathcal{A}(A))$ the smallest algebra containing all polynomials in $A$ and closed in the weak operator (respectively, weak*) topology.

We denote by $x \cdot y$ the rank-one operator defined by $(x \cdot y) u=(u, y) x$. If $\mathcal{S}$ is a linear space in $\mathcal{L}(H)$, then the weak*-continuous linear functionals on $\mathcal{S}$ are exactly those which take the form

$$
\phi(A)=\sum_{n=1}^{\infty}\left(A x_{n}, y_{n}\right)=\operatorname{tr}\left(A \sum_{n=1}^{\infty}\left(x_{n} \cdot y_{n}\right)\right), \quad A \in \mathcal{S}
$$

where $\left\{x_{n}\right\}$ and $\left\{y_{n}\right\}$ are square-summable sequences from $H$. Moreover, the sequences $\left\{x_{n}\right\}$ and $\left\{y_{n}\right\}$ can be chosen so that $\|\phi\|^{2}=\sum\left\|x_{n}\right\|^{2}=\sum\left\|y_{n}\right\|^{2}$. Therefore every trace-class operator $t$ can be represented as $t=\sum_{n=1}^{\infty}\left(x_{n} \cdot y_{n}\right)$ with square-summable sequences $\left\{x_{n}\right\}$ and $\left\{y_{n}\right\}$ from $H$. We denote by $\mathcal{S}_{\perp}$ the pre-annihilator of $\mathcal{S}$ in $\tau c(H)$.

The proof of the next lemma follows easily from the characterization of trace-class operators and we will leave out the details.

Lemma 2.1. Let $t \in \tau c(H)$ and suppose $\left\{p_{\jmath}\right\}_{\jmath \in \mathcal{J}}$ is a mutually orthogonal family of projections on $H$. Then $d=\sum_{\jmath \in \mathcal{J}} p_{\jmath} t p_{\jmath}$ is also in the trace class and $\|d\|_{1} \leqslant\|t\|_{1}$.

The operator $d$ in the above lemma is the "diagonal part" of $t$ when $t$ is represented as a block matrix with respect to the projections $\left\{p_{\jmath}\right\}$.

Next we review some basic properties of reflexivity.

The reflexive closure of a linear manifold $\mathcal{S}$ in $\mathcal{L}(H)$ is defined by

$$
\operatorname{Ref} \mathcal{S}=\{A \in \mathcal{L}(H): A x \in \overline{\mathcal{S} x} \text { for all } x \in H\} .
$$


The subspace $\mathcal{S}$ is said to be reflexive if $\operatorname{Ref} \mathcal{S}=\mathcal{S}$. When $\mathcal{S}$ is an algebra with identity, this definition coincides with the definition of reflexive algebra given in the introduction. Observe that an operator $B \in \mathcal{L}(H)$ belongs to $\operatorname{Ref} \mathcal{S}$ if and only if for all vectors $x, y \in H,(A x, y)=0$ for all $A \in \mathcal{S}$ implies that $(B x, y)=0$.

The following direct consequence of the definition will be useful later.

Proposition 2.2. Suppose $u$ is an isometry mapping $K$ to $H$. Then $u^{*} \operatorname{Ref}(\mathcal{S}) u$ $\subset \operatorname{Ref}\left(u^{*} \mathcal{S} u\right)$.

Further properties of reflexive closure can be found in [1].

Next we discuss the celebrated property $\left(\mathbb{A}_{1}\right)$ that has played an integral role in invariant subspace and reflexivity theories. A linear manifold $\mathcal{S}$ in $\mathcal{L}(H)$ is said to have property $\left(\mathbb{A}_{1}\right)$ if $\mathcal{S}_{\perp}+\mathcal{F}_{1}=\tau c$, that is, every weak*-continuous linear functional on $\mathcal{S}$ can be represented by a rank-one operator. (In [1], a manifold enjoying property $\left(\mathbb{A}_{1}\right)$ is referred to as elementary.) Equivalently, $\mathcal{S}$ has property $\left(\mathbb{A}_{1}\right)$, if given a weak*-continuous linear functional $\phi$ on $\mathcal{S}$, there are vectors $x, y \in H$ such that $\phi(A)=(A x, y)$ for all $A \in \mathcal{S}$. If in addition the norms $x$ and $y$ can be bounded by a universal constant $\mathrm{r}$ (independent of $\phi$ ), i.e. $\|x\|,\|y\| \leq s\|\phi\|^{1 / 2}$, for all $s>r$, then we say $\mathcal{S}$ has property $\left(\mathbb{A}_{1}(r)\right)$. As noted in [8, Proposition 59.2], the weak and weak* closures of any linear manifold having property $\left(\mathbb{A}_{1}(r)\right)$ coincide. In particular, for any operator $A \in \mathcal{L}(H)$ if the algebra $\mathcal{A}(A)$ has property $\mathbb{A}_{1}(r)$ then $\mathcal{A}(A)=\mathcal{W}(A)$. We say $\mathcal{S}$ has property $\left(\mathbb{A}_{\aleph_{0}}\right)$ if for any given doubly indexed sequence $\phi_{i j}$ of weak*-continuous linear functionals on $\mathcal{S}$, there are sequences $\left\{x_{n}\right\}$ and $\left\{y_{n}\right\}$ in $H$ such that $\phi_{i j}(A)=\left(A x_{i}, x_{j}\right)$ for all $A \in \mathcal{S}$. Further details and applications concerning $\left(\mathbb{A}_{1}\right)$ and related properties can be found in [6].

In general, reflexivity is not hereditary with respect to inclusion for linear manifolds of $\mathcal{L}(H)$, nor is it preserved when direct sums are formed. The following propositions will be our basic tools for establishing reflexivity in the sequel. The first one appeared in [17] (see also [2, Proposition 1.7]).

Proposition 2.3. Let $\mathcal{S}$ be a linear manifold in $\mathcal{L}(H)$.

1. If $\mathcal{S}$ has property $\left(\mathbb{A}_{1}\right)$ (resp. $\left.\left(\mathbb{A}_{1}(r)\right)\right)$, then every weak ${ }^{*}$-closed subspace of $\mathcal{S}$ also has property $\left(\mathbb{A}_{1}\right)$ (resp. $\left.\left(\mathbb{A}_{1}(r)\right)\right)$.

2. Suppose $\mathcal{S}$ is reflexive. Then every weak ${ }^{*}$-closed subspace of $\mathcal{S}$ is reflexive if and only if $\mathcal{S}$ has property $\left(\mathbb{A}_{1}\right)$.

[14, Theorem 3.8 and 4.1] give us the following properties of direct sums.

Proposition 2.4. Let $\mathcal{J}$ be an index set and $\left\{H_{i}\right\}_{i \in \mathcal{J}}$ be a collection of Hilbert spaces and suppose $A_{i} \in \mathcal{L}\left(H_{i}\right)$. If there exists an $r \geq 1$, such that for each $i \in \mathcal{J}, \mathcal{W}\left(A_{i}\right)$ has property $\left(\mathbb{A}_{1}(r)\right)$, then $\oplus_{i \in \mathcal{J}} \mathcal{W}\left(A_{i}\right)$ and $\mathcal{W}\left(\oplus_{i \in \mathcal{J}} A_{i}\right)$ also have property $\left(\mathbb{A}_{1}(r)\right)$. If in addition each $A_{i}$ is reflexive, then $\oplus_{i \in \mathcal{J}} \mathcal{W}\left(A_{i}\right)$ and $\mathcal{W}\left(\oplus_{i \in \mathcal{J}} A_{i}\right)$ are reflexive as well.

We write $a_{s}$ for the unilateral shift acting on the Hilbert space $l_{+}^{2}$ defined by $a_{s}\left(x_{0}, x_{1}, \ldots\right)=\left(0, x_{0}, x_{1}, \ldots\right)$. The backward shift $a_{s}^{*}$ satisfies $a_{s}^{*}\left(x_{0}, x_{1}, \ldots\right)=\left(x_{1}, x_{2}, \ldots\right)$. Let $H_{k}$ be the $k$-dimensional subspace 
of $l_{+}^{2}$ corresponding to the first $k$ components of $l_{+}^{2}$, which is an invariant subspace for $a^{*}$. The truncated shift $a_{k}$ is simply $a_{s}$ compressed to $H_{k}$, i.e., $a_{k}=P_{k} a_{s} \mid H_{k}$, where $P_{k}$ is orthogonal projection onto $H_{k}$. The matrix representation of a truncated shift is a nilpotent Jordan block of size $k$. Note also that each truncated shift $a_{k}$ is unitarily equivalent to its adjoint $a_{k}^{*}$. It is well known that $a_{s}, a_{s}^{*}$ and $a_{k}$ all have property $\left(\mathbb{A}_{1}(1)\right)$, though for somewhat different reasons. See [8, Proposition 60.5] and [6, Theorem 2.06]) for details.

\section{Consistent Operators}

An individual operator $A$ is said to be reflexive, have Property $\left(\mathbb{A}_{1}\right)$, etc. if the algebra $\mathcal{W}(A)$ enjoys the corresponding property.

Let $\left\{S_{i}\right\}$ be a family of operator spaces. It follows from the definition that $\operatorname{Ref}\left(\oplus S_{i}\right)=\oplus\left(\operatorname{Ref} S_{i}\right)$. In particular, direct sums of reflexive operator spaces are again reflexive. In general however the direct sum of reflexive operators need not be reflexive. The first counterexample was given by Larson and Wogen [16]. There are many special cases when direct sums do preserve reflexivity of individual operators. For example, if projection on the first summand belongs to $\mathcal{W}(a \oplus b)$, then $\mathcal{W}(a \oplus b)=\mathcal{W}(a) \oplus \mathcal{W}(b)$ and thus individual reflexivity of $a$ and $b$ implies reflexivity of $a \oplus b$ as well.

Like many papers before it, a good deal of our motivation comes from the Deddens-Fillmore result [11]. Let $a \in M_{n}$. Since similarity preserves reflexivity, we may as well assume $a$ to be in (upper) Jordan canonical form. Projections corresponding to the various generalized eigenspaces of $a$ belong to $\mathcal{W}(a)$ and thus the last paragraph reduces the study of reflexivity of members of $M_{n}$ to the study of reflexivity for nilpotents.

One of the advantages of shifts (of any size) is that the matrices of their powers are supported on distinct diagonals and thus do not interfere with each other. Nevertheless, the simplest shifts $-a_{n}$ with $1<n<\infty$ - are not reflexive. Indeed all invariant subspaces of $a_{n}$ take the form span $\left\{e_{1}, \ldots, e_{k}\right\}$ for $1 \leq k \leq n$ and thus all upper-triangular matrices belong to Ref $a_{n}$.

The situation changes when we look at $a_{n} \oplus a_{n}$ acting on $\mathbb{C}^{n} \oplus \mathbb{C}^{n}$. Set $G:=\mathcal{W}\left(a_{n} \oplus a_{n}\right)$. Looking at the most obvious invariant subspaces we see that every member of $\operatorname{Ref} G$ takes the form $a \oplus b$ where $a$ and $b$ are uppertriangular. So fix $a \oplus b$ in Ref $G$. By looking at actions on vectors of the form $e_{i} \oplus e_{j}$ we can get a lot more information. In particular, for any $g \in G$, we have $g\left(e_{j} \oplus e_{j}\right)$ takes the form $x \oplus x$; thus the same must be true for each member of $\operatorname{Ref} G$ and we see that $a=b$. But now, considering action on $e_{j} \oplus e_{n}$, we see that the $j$ 'th column of $a$ must coincide with the top part of the $n$th column of $b$ and thus with the top part of its own $n$th column. We have now gathered enough information to conclude that a belongs to $\mathcal{W}\left(a_{n}\right)$ and thus $a_{n} \oplus a_{n}$ is indeed reflexive.

When we consider $a_{m} \oplus a_{n}$ with $m<n$, the above procedure only gives us partial information - every member of $\mathcal{W}\left(a_{m} \oplus a_{n}\right)$ takes the form $a \oplus b$ where $a$ coincides with the upper $m$ by $m$ corner of $b$; the same must therefore 
be true for every member of $\operatorname{Ref}\left(a_{m} \oplus a_{n}\right)$. In [11], this approach leads to the conclusion that $a_{m} \oplus a_{n}$ is reflexive if $|m-n| \leq 1$.

The notion of "consistent operator" will enable us to efficiently exploit this idea of finding copies of one direct summand of an operator inside another direct summand.

Let $\left\{H_{\imath}\right\}_{\imath \in \mathcal{J}}$ be a family of Hilbert spaces indexed by a partially ordered countable set $(\mathcal{J}, \leqslant)$ and suppose that for each $\imath, \jmath \in \mathcal{J}$ such that $\imath \leqslant \jmath$ we are given an isometry $v_{\imath \jmath}: H_{\imath} \rightarrow H_{\jmath}$.

Set $\mathcal{H} \equiv \oplus_{\imath \in \mathcal{J}} H_{\imath}$ and write $\left\{p_{\imath}\right\}$ for the canonical projection operators on $\mathcal{H}$ associated with this direct sum. Operators on $\mathcal{H}$ of the form $A=\oplus_{\imath \in \mathcal{J}} A_{\imath} \in \mathcal{L}(\mathcal{H})$ are said to be decomposable with respect to the family $\left\{H_{\imath}\right\}_{\imath \in \mathcal{J}}$ (and just decomposable when the context is clear); the collection of decomposable operators is denoted by $\mathcal{D}$.

Definition 3.1. A decomposable operator $A=\oplus_{\imath \in \mathcal{J}} A_{\imath}$ associated with the Hilbert space family $\left\{H_{\imath}\right\}_{\imath \in \mathcal{J}}$ equipped with isometries $v_{\imath \jmath}: H_{\imath} \rightarrow H_{\jmath}$ is said to be consistent if

$$
v_{\imath \jmath}^{*} A_{\jmath} v_{\imath \jmath}=A_{\imath}
$$

whenever $\imath \leqslant \jmath$. The collection of consistent operators is denoted by $\mathcal{C}$.

It should be emphasized that $\mathcal{C}$ depends on the direct sum decomposition $\mathcal{H} \equiv \oplus_{\imath \in \mathcal{J}} H_{\imath}$, the order with which $\mathcal{J}$ is equipped, and the choice of isometries $\left(v_{\imath \jmath}\right)$. This dependence will not be indicated notationally however as the relevant parameters should be clear from context.

For the applications, it will often suffice to take $\mathcal{J}$ to be $\mathbb{N}$, the positive integers equipped with the standard order. When all the $\left\{H_{\imath}\right\}$ coincide and each $v_{\imath, \jmath}$ is the identity, then the set $\mathcal{C}$ reduces to the infinite ampliation $\mathcal{L}\left(H_{1}\right)^{(\infty)}$; this is a von Neumann algebra well-known to be reflexive and to have property $\left(\mathbb{A}_{\aleph_{0}}\right)$.

The motivating example for the current paper, however, takes $H_{n}=\mathbb{C}^{n}$ for each $n \in \mathbb{N}$, the maps $v_{\imath}$ being the standard embeddings. In this case, members of $\mathcal{C}$ are direct sums $\oplus_{n \in \mathbb{N}} A_{n}$ where each $A_{n}$ reappears as the upperleft corner of $A_{n+1}$. As is typical, this collection of consistent operators fails to be an algebra. The following proposition shows however that $\mathcal{C}$ is always reflexive; this proof actually has nothing to do with the order structure on $\mathcal{J}$.

Proposition 3.2. The space $\mathcal{C}$ of consistent operators is reflexive.

Proof. Suppose $B \in \operatorname{Ref} \mathcal{C}$. Since the collection of decomposable operators is a von Neumann algebra, we at least know that $B=\oplus_{\imath \in \mathcal{J}} B_{\imath}$ is decomposable. To see that $B$ is consistent, take $\imath, \jmath \in \mathcal{J}$ such that $\imath \leqslant \mathcal{\jmath}, \imath \neq \jmath$ and define $u: H_{\imath} \oplus H_{\imath} \rightarrow \mathcal{H}$ by $u(x \oplus y)=x \oplus v_{\imath} y \oplus 0$, where the last 0 means that all components except $\imath, \jmath$ are equal to 0 . For each decomposable operator $A=\oplus_{\imath \in \mathcal{J}} A_{\imath}$ we have $u^{*} A u=A_{\imath} \oplus v_{\imath \jmath}^{*} A_{\jmath} v_{\imath \jmath}$. By the definition of $\mathcal{C}$, these two direct summands are equal, so

$$
u^{*} \mathcal{C} u \subseteq\left\{C \oplus C: C \in \mathcal{L}\left(H_{\imath}\right)\right\}=\mathcal{L}\left(H_{\imath}\right)^{(2)},
$$


which is reflexive. On the other hand, Proposition 2.2 tells us that $u^{*} B u \in$ $\operatorname{Ref}\left(u^{*} \mathcal{C} u\right)$. It follows that $B_{\imath} \oplus v_{\imath \jmath}^{*} B_{\jmath} v_{\imath \jmath} \in \mathcal{L}\left(H_{\imath}\right)^{(2)}$, whence $B_{\imath}=v_{\imath \jmath}^{*} B_{\jmath} v_{\imath \jmath}$. Hence $B \in \mathcal{C}$.

We now proceed to the core technical argument of the paper.

Proposition 3.3. Let $\mathcal{J}$ be a linearly ordered countable set which does not have a largest element. Then the space $\mathcal{C}$ of consistent operators on $\mathcal{H}=\oplus_{\imath \in \mathcal{J}} H_{\imath}$ has property $\left(\mathbb{A}_{1}(1)\right)$.

Proof. Let $t \in \tau c(\mathcal{H})$; we must find $f \in \mathcal{F}_{1}$ satisfying $\|f\|_{1} \leqslant\|t\|_{1}$ and $t-f \in \mathcal{C}_{\perp}$.

We accomplish this by making several reductions. In view of Proposition 2.1 , we know that the decomposable operator $\sum_{\imath \in \mathcal{J}} p_{\imath} t p_{\imath}$ belongs to $\tau c$ and that its trace norm cannot exceed the trace norm of $t$. It is also easy to check that $t-\sum_{\imath \in \mathcal{J}} p_{\imath} t p_{\imath}$ is orthogonal to all of $\mathcal{D}$. This shows there is no loss of generality in assuming that $t=\oplus_{\imath \in \mathcal{J}} t_{\imath}$ is itself decomposable. Our main task is to reduce to the simpler situation in which each $t_{\imath}$ has rank one. Apply the structure of trace class operators to write $t_{\imath}=\sum_{j \in \mathbb{N}} t_{\imath, j}$ for each $\imath$ with $\sum_{\imath \in \mathcal{J}} \sum_{j \in \mathbb{N}}\left\|t_{\imath, j}\right\|_{1}=\|t\|_{1}$.

Next apply the absence of a largest element in $\mathcal{J}$ to recursively construct an injective map $\alpha: \mathcal{J} \times \mathbb{N} \rightarrow \mathcal{J}$ which satisfies $\alpha(\imath, j) \geqslant \imath$ for all $\imath \in \mathcal{J}$ and $j \in \mathbb{N}$.

For each $\imath \in \mathcal{J}$ and $j \in \mathbb{N}$ set $s_{\alpha(\imath, j)}=v_{\imath \alpha(\imath, j)} t_{\imath, j} v_{\imath \alpha(\imath, j)}^{*}$. Thus $s_{\alpha(\imath, j)}$ is an operator on $H_{\alpha(\imath, j)}$ with trace norm no larger than that of $t_{\imath, j}$. Moreover, for any consistent operator $A=\oplus_{\imath \in \mathcal{J}} A_{\imath}$, we have

$$
\left\langle A_{\imath}, t_{\imath, j}\right\rangle=\left\langle v_{\imath \alpha(\imath, j)}^{*} A_{\alpha(\imath, j)} v_{\imath \alpha(\imath, j)}, t_{\alpha(\imath, j)}\right\rangle=\left\langle A_{\alpha(\imath, j)}, s_{\alpha(\imath, j)}\right\rangle
$$

for all $\imath \in \mathcal{J}$ and $j \in \mathbb{N}$. It follows that

$$
\begin{aligned}
\langle A, t\rangle & =\sum_{\imath \in \mathcal{J}} \sum_{j \in \mathbb{N}}\left\langle A_{\imath}, t_{\imath, j}\right\rangle \\
& =\sum_{\imath \in \mathcal{J}} \sum_{j \in \mathbb{N}}\left\langle A_{\alpha(\imath, j)}, s_{\alpha(\imath, j)} \geq \sum_{\iota \in \operatorname{Range}(\alpha)}\left\langle A_{\iota}, s_{\iota}\right\rangle .\right.
\end{aligned}
$$

Set $s_{\iota}:=0$ when $\iota$ is not in the range of $\alpha$ and take $s:=\oplus_{\iota \in \mathcal{J}} s_{\iota}$. Then $\|s\|_{1} \leqslant\|t\|_{1}$, and the preceding equation shows that $t-s \perp \mathcal{C}$. Replacing $t$ by $s$ if necessary, we have thus successfully completed our reduction to the case where the $\left\{t_{\imath}\right\}$ all have rank one.

For each $\iota \in \mathcal{J}$, choose vectors $x_{\iota}, y_{\iota} \in H_{\iota}$ with $\left\|x_{\iota}\right\|=\left\|y_{\iota}\right\|=\sqrt{\left\|t_{\iota}\right\|_{1}}$. Then $f=\left(\oplus_{\iota \in \mathcal{J}} x_{\iota}\right) \cdot\left(\oplus_{\iota \in \mathcal{J}} y_{\iota}\right)$ is a rank one operator on $\mathcal{H}$ with the same diagonal and trace norm as $t$. In particular, $t-f \perp \mathcal{C}$ and the proof is complete.

Combining Propositions 2.3, 3.2, and 3.3 yields the main result of this section.

Corollary 3.4. Let $\mathcal{J}$ be a linearly ordered countable set which does not have a largest element. Then each weak*-closed subspace of the space $\mathcal{C}$ of consistent operators is reflexive and enjoys property $\left(\mathbb{A}_{1}(1)\right)$. 
The linear ordering hypothesis of the last two results can be weakened; it suffices to assume that $(\mathcal{J}, \leqslant)$ is a directed set. Simple examples like $\mathcal{W}\left(a_{1} \oplus\right.$ $a_{3}$ ) however, show that the absence of a largest member in $\mathcal{J}$ is a crucial hypothesis. We do have a partial complementary result.

Proposition 3.5. Let $\mathcal{J}$ be a partially ordered set having a largest element $\imath_{0}$ and suppose $\mathcal{T}$ is a weak*-closed subspace of consistent operators. If the restriction $\mathcal{T}_{\imath_{0}}:=\left.\mathcal{T}\right|_{H_{i_{0}}}$ is reflexive, then $\mathcal{T}$ is reflexive as well.

Proof. Let $B \in \operatorname{Ref} \mathcal{T}$. As in Proposition 3.2, we obtain the decomposition $B=\oplus_{\imath \in \mathcal{J}} B_{\imath}$. From reflexivity of $\left.\mathcal{T}\right|_{H_{\iota_{0}}}$ we find $A=\oplus_{\imath \in \mathcal{J}} A_{\imath} \in \mathcal{T}$ with $B_{\iota_{0}}=A_{\imath_{0}}$. In particular, $A$ and $B$ both belong to Ref $\mathcal{C}$. Since the space $\mathcal{C}$ of consistent operators is reflexive, we conclude that

$$
A_{\imath}=v_{\imath \imath_{0}}^{*} A_{\imath_{0}} v_{\imath \imath_{0}}=v_{\imath \imath_{0}}^{*} B_{\imath_{0}} v_{\imath \iota_{0}}=B_{\imath}
$$

for every $\imath \in \mathcal{J}$. Thus $B=A \in \mathcal{T}$ and the proof is complete.

For completeness, we also record the following consequence of Proposition 3.3 .

Theorem 3.6. Let $\mathcal{J}$ be a linearly ordered countable set which does not have a largest element. Then the space $\mathcal{C}$ of consistent operators on $\mathcal{H}=\oplus_{\imath} \in \mathcal{J} H_{\imath}$ is reflexive and has property $\mathbb{A}_{\aleph_{0}}$.

Proof. We need only establish the second assertion. This is equivalent to the assertion that $\mathcal{C}(\mathcal{H}) \otimes \mathcal{L}\left(\ell_{2}\right)$ has property $\mathbb{A}_{1}$. Continue to denote the isom-

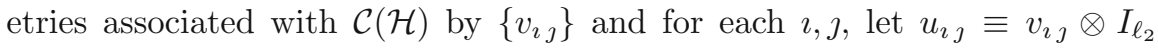
map $H_{\imath} \otimes \ell_{2}$ into $H_{\jmath} \otimes \ell_{2}$. Then $\mathcal{C}(\mathcal{H}) \otimes \mathcal{L}\left(\ell_{2}\right)$ is precisely the collection of consistent operators associated with the isometries $\left\{u_{\imath, \jmath}\right\}$ whence the proof is completed by appealing to Corollary 3.4.

\section{Reflexivity of Power Partial Isometries}

Recall that an operator $V \in \mathcal{L}(H)$ is a partial isometry if $V^{*} V$ is an orthogonal projection or equivalently $V V^{*} V=V$. An operator $S$ is a power partial isometry if $S^{n}$ is a partial isometry for all $n \in \mathbb{N}$.

Examples of power partial isometries include unitary operators, the unilateral shift and its adjoint, and the truncated shifts of each order. It follows that all direct sums of such operators remain power partial isometries. In their paper [15], Halmos and Wallen proved the following converse.

Proposition 4.1. Let $S$ be a power partial isometry acting on a Hilbert space $H$. Then $S$ enjoys a unique orthogonal decomposition

$$
S=U \oplus F \oplus G \oplus T
$$

where $U$ is unitary, $F$ is a direct sum of forward unilateral shifts, $G$ is a direct sum of backward shifts, and $T$ is a direct sum of truncated shifts.

Any of $U, F, G, T$ may be absent and the last three may themselves involve infinitely many direct summands.

Proposition 4.2. Every power partial isometry has Property $\left(\mathbb{A}_{1}(1)\right)$. 
Proof. This is implicit for normal operators and for the forward (and hence backward) shift in [18]; it is recorded for truncated shifts in Proposition 1.21(iv) of Bercovici's monograph [4]. In view of Proposition 2.3, all direct sums of such operators continue to enjoy Property $\left(\mathbb{A}_{1}(1)\right)$ whence appeal to the last result completes the argument.

Lemma 4.3. Suppose $1 \leq m \leq n \leq \infty$. Then there is an isometry $v:=v_{m, n}$ : $\mathbb{C}^{n} \rightarrow \mathbb{C}^{m}$ satisfying $v^{*} a_{n}^{k} v=a_{m}^{k}$ for each $k>0$.

Proof. We assume $n<\infty$; cosmetic changes adapt the argument to the forward shift $a_{\infty}:=a_{s}$, its adjoint, and the bilateral shift.

Write $e_{1}, \ldots, e_{m}$ for the standard basis of $\mathcal{C}^{m}$ and $f_{1}, \ldots, f_{m}$ for the standard basis of $\mathcal{C}^{n}$. Define $v e_{j}:=f_{j}$ for $1 \leq j \leq m$ and compute.

The consistency concept was invented to set up the following proof.

Proposition 4.4. Suppose $T$ is a direct sum of truncated shifts acting on finitedimensional Hilbert spaces. If $T$ is not nilpotent, then it is reflexive.

Proof. We order the family $\mathcal{J}$ of underlying Hilbert spaces by first putting in the (possibly infinitely many) copies of $\mathbb{C}^{1}$ followed by the copies of $\mathbb{C}^{2}$, etcetera. This is a linear order on $\mathcal{J}$; for the connecting partial isometries, we use the injections provided by Lemma 4.3. Since $T$ is non-nilpotent, our partial order does not admit a largest element, and thus Propositions 3.2 and 3.3 assure us that the corresponding space $\mathcal{C}$ of consistent operators is reflexive and elementary. Since Lemma 4.3 guarantees $\mathcal{W}(T) \subset \mathcal{C}$, we conclude that $\mathcal{W}(T)$ is reflexive as well.

It is worthwhile understanding why the proof of Proposition 4.4 fails for nilpotent $T$. After all, it is still the case that $\mathcal{W}(T)$ is elementary and Proposition 3.2 still tells us that $\mathcal{C}$ is reflexive. The problem is that $\mathcal{C}$ may not be elementary and thus we have no basis to apply Proposition 2.3.

In fact, the corresponding result in the nilpotent case is well-known.

Proposition 4.5. The following are equivalent for nilpotent $T$.

1. $T$ is reflexive.

2. The sizes of the two largest blocks in the Jordan form of $T$ differ by at most one.

3. Each $B \in \mathcal{W}(T)$ of rank 2 generates a one-dimensional ideal.

Proof. The equivalence of (1) and (2) is carried out by Hadwin in [13]. (The full force of (2) implies (1) also follows from the 2-block case by applying Proposition 3.5 with the maximal element of $\mathcal{J}$ corresponding to the direct sum of the two largest blocks of $T$ ).

The equivalence of (2) and (3) is addressed in [3].

Theorem 4.6. Let $S$ be a power partial isometry with absolutely continuous unitary part. Then the following are equivalent:

1. $S$ is reflexive.

2. Each $B \in \mathcal{W}(S)$ of rank 2 generates a one-dimensional ideal. 
Proof. Decompose $S=U \oplus F \oplus G \oplus T$ as in Proposition 4.2. If only $T$ is present, the situation is covered by the last two results.

If $F$ or $G$ is present, then reflexivity of $F \oplus G \oplus T$ follows from the main result of Wu's paper [19]. (When precisely one of $F, G$ is present, one could instead apply Proposition 3.5 above.) On the other hand, the presence of $F$ and/or $G$ means there are no non-trivial finite-rank operators in $\mathcal{W}(S)$ so $(2)$ holds vacuously.

Now suppose we also allow a direct summand $U$ with absolutely continuous scalar spectral measure $\mu$. If Lebesgue measure $m$ is absolutely continuous with respect to $\mu$, then $U$ has a bilateral shift direct summand and thus $U \oplus T$ and hence $S$ are reflexive regardless of the values of $F, G, T$. Also the presence of $U$ excludes non-trivial finite-rank members of $\mathcal{W}(S)$ so again (1) and (2) both hold.

Finally suppose $\mu$ is supported on a proper closed subset of the unit circle. Again, if $F$ or $G$ is present, then (1) and (2) will simultaneously hold. On the other hand, if $S=U \oplus T$, we can apply Mergelyan's Theorem to show that $\mathcal{W}(S)=\mathcal{W}(U) \oplus \mathcal{W}(T)$, so conditions (1) and (2) respectively reduce to the corresponding conditions for $\mathcal{W}(T)$.

It remains to examine the reflexivity effect of a possible singular unitary direct summand. The characterization of the last theorem does not hold if e.g. $S:=\left[\begin{array}{cc}1 & 0 \\ 0 & -1\end{array}\right] \oplus 0$, but we can hope for the following.

Conjecture 4.7. Suppose $S=U \oplus F \oplus G \oplus T$ as in Proposition 4.1. Then in order for $S$ to be reflexive, it is necessary and sufficient that

1. F or $G$ is non-trivial or

2. $T$ is non-nilpotent or $T$ is nilpotent and the sizes of its two largest blocks differ by at most one or

3. Lebesgue measure on the circle is absolutely continuous with respect to the scalar spectral measure of $U$.

We already have sufficiency. To establish necessity, we may assume $F, G$ absent, $T$ non-reflexive (in particular nilpotent), and write $U_{a}, U_{s}$ for the absolutely continuous and singular parts of $U$ respectively. It would then suffice to show $\mathcal{W}\left(U_{s} \oplus\left(U_{a} \oplus T\right)\right)$ splits as the direct sum of $\mathcal{W}\left(U_{s}\right)$ and $\mathcal{W}\left(U_{a} \oplus T\right)$, but we have been unable to coax this from J.B. Conway's analysis of splitting in [9].

\section{Related Work}

Remark 5.1. The motivating discussion of the Deddens-Fillmore proof at the beginning of Sect. 3 can be made more conceptual by use of separating vectors. See, for example, Lifeng Ding's paper [12].

Remark 5.2. The truncated shifts $\left(a_{n}\right)$ are a special case of the Jordan blocks $S(\theta)$ associated with inner functions and studied in [4]. Jordan operators are direct sums of these corresponding to linearly ordered families of 
inner functions, the order relation being divisibility. These provide a set of models for operators of class $C_{0}$. In particular, Theorem 4.1 .23 of [4] is a far-reaching generalization of Proposition $4.5(1) \Leftrightarrow(2)$ above. The operators of Proposition 4.4, however, are not of class $C_{0}$ as there is no inner function simultaneously divisible by arbitrarily large powers of $z$. The function-theoretic and quasi-similarity considerations of [4] are much deeper than those of the present paper, but it seemed worthwhile to the present authors to record the purely order-theoretic content of Corollary 3.4.

Remark 5.3. As mentioned in the abstract, our definition of consistency is motivated by von Neumann algebra theory. The set $\mathcal{D}$ of decomposable operators relative to the given decomposition $H=\oplus_{i \in \mathcal{J}} H_{i}$ do indeed form a von Neumann algebra and our use of the term "decomposable" is borrowed from that subject. The projections $\left\{p_{j}\right\}$ corresponding to this decomposition belong to the center of $\mathcal{D}$. Each isometry $v_{i, j}$ of Definition 3.1 extends to a partial isometry $u=u_{i, j}$ on all of $H$ by sending $\operatorname{kernel}\left(p_{i}\right)$ to zero. Then $u^{*} u=p_{i}$ while $u u^{*} \leq p_{j}$, which is a way of saying $p_{i}$ is smaller than $p_{j}$ as expected from $i \leq j$. On the other hand, in the present paper, the pregiven partial isometries are used to define the class of consistent operators rather than the other way around.

Remark 5.4. The operators covered by Proposition 4.4 can be thought of as weighted shifts involving infinitely many zero weights, all other weights being 1, there being arbitrarily long intervals between successive zeros. It is possible to allow weights other than 0 and 1 by replacing Eq. 3.1 by

$$
c_{\imath \jmath} A_{\jmath} d_{\imath \jmath}=A_{\imath},
$$

where $c_{i, j} d_{i, j}=p_{i}$ and $d_{i, j} c_{i, j} \leq p_{j}$ for each $i \leq j$. If the norms of the operators $c_{i, j}, d_{i, j}$ are uniformly bounded, then the resulting space $\mathcal{C}$ of consistent operators will have Property $\left(\mathbb{A}_{1}(r)\right)$ for some $r$. On the other hand, Thereom 5.10 of [14] can be modified to construct a weighted shift with arbitrarily long intervals between successive zeros which does not enjoy Property $\left(\mathbb{A}_{1}\right)$.

A different approach to weighted shifts of this type in Section 4.3 of Bindner's dissertation [7] shows that even the non-elementary ones are still reflexive; his proof efficiently exploits function theory and Schur products.

Remark 5.5. Finally we mention H. Bercovici's paper [5]. Suppose $\mathcal{M}$ is a weakly closed operator space whose commutant contains two isometries having orthogonal ranges. Theorem 4.3 of the paper implies that $\mathcal{M}$ enjoys Property $\left(\mathbb{A}_{1}\right)$, while the following Corollary 4.4 implies that $\mathcal{M}$ is also reflexive. This does not cover the results of the present paper since the consistent operators of Definition 3.1 seldom commute with the isometries used to define them.

Open Access. This article is distributed under the terms of the Creative Commons Attribution Noncommercial License which permits any noncommercial use, distribution, and reproduction in any medium, provided the original author(s) and source are credited. 


\section{References}

[1] Azoff, E.A.: On finite rank operators and preannihilators. Mem. Am. Math. Soc. 357 (1986)

[2] Azoff, E.A., Ptak, M.: A dichotomy for linear spaces of Toeplitz operators. J. Funct. Anal. 156, 411-428 (1998)

[3] Azoff, E.A., Ptak, M.: On rank two linear transformations and reflexivity. J. Lond. Math. 53, 383-396 (1996)

[4] Bercovici, H.: Operator theory and aritmetic in $H^{\infty}$. In: Mathematical Surveys and Monographs No. 26. American Mathematical Society, Providence (1988)

[5] Bercovici, H.: Hyper-reflexivity and the factorization of linear functionals. J. Funct. Anal. 158, 242-252 (1988)

[6] Bercovici, H., Foiaş, C., Pearcy, C.: Dual Algebras with Applications to Invariant Subspaces and Dilation Theory. CBMS Regional Conference Series in Mathematics, vol. 56, American Mathematical Society, Providence (1985)

[7] Bindner, D.: On the space spanned by the powers of an operator and its adjoint. Dissertation, University of Georgia (2001)

[8] Conway, J.B.: A course in operator theory. American Mathematical Society, Providence (2000)

[9] Conway, J.B.: The direct sum of normal operators. Indiana Univ. Math J. 26, 277-289 (1977)

[10] Deddens, J.A.: Every isometry is reflexive. Proc. Amer. Math. Soc. 28, 509-512 (1971)

[11] Deddens, J.A., Fillmore, P.A.: Reflexive linear transformations. Linear Algebra Appl. 10, 89-93 (1975)

[12] Ding, L.: Separating vectors and reflexivity. Linear Algebra Appl. 174, 37-52 (1992)

[13] Hadwin, D.: Algebraically reflexive linear transformations. Linear Multilinear Algebra 14, 225-233 (1983)

[14] Hadwin, D., Nordgren, E.A.: Subalgebras of reflexive algebras. J. Oper. Theory 7, 3-23 (1982)

[15] Halmos, P.R., Wallen, L.J.: Powers of partial isometries. J. Math. Mech. 19, 657-663 (1970)

[16] Larson, D.R., Wogen, W.R.: Reflexivity properties of $T \oplus 0$. J. Funct. Anal. 92, 448-467 (1999)

[17] Loginov, A.I., Sulman, V.I.: On hereditary and intermediate reflexivity of $W^{*}$ algebras. Soviet Math. Dokl. 14, 1473-1476 (1973)

[18] Sarason, D.: Invariant subspaces and unstarred operator algebras. Pacific J. Math. 17, 511-517 (1966)

[19] Wu, P.Y.: Contractions with a unilateral shift summand are reflexive. Integral Equ. Oper. Theory 7, 899-903 (1984)

Edward A. Azoff

Department of Mathematics

University of Georgia

Athens, GA 30602

USA

e-mail: azoff@math.uga . edu 


\author{
Wing Suet Li \\ Department of Mathematics \\ Georgia Institute of Technology \\ Atlanta, GA 30332 \\ USA \\ e-mail: li@math.gatech.edu \\ Mostafa Mbekhta \\ UFR de Mathematiques UMR-CNRS 8524 \\ Universite Lille 1 \\ 59655 Villeneuve d'Ascq \\ France \\ e-mail: mbekhta@agat.uviv-lille1.fr \\ Marek Ptak $(\bowtie)$ \\ Institute of Mathematics \\ University of Agriculture \\ ul. Balicka 253C \\ 30-198 Kraków \\ Poland \\ e-mail: rmptak@cyf-kr.edu.pl \\ and \\ Institute of Mathematics \\ Pedagogical University \\ ul. Podchorych 2 \\ 30-084 Kraków \\ Poland
}

Received: September 14, 2010.

Revised: June 21, 2011. 